\title{
Research on Cognitive Function of Abstracts based on English Linguistics
}

\author{
Donghui Li \\ Dalian Art College, Dalian, Liaoning, 116600
}

Keywords: Cognitive Function, English Linguistics, English Abstracts

\begin{abstract}
As an important branch of academic papers, linguistic academic papers occupy a place in cultural communication, and the coherence of abstract texts has an important influence on the language features and functions of texts. Scholars have conducted research and analysis from many angles, but from the perspective of cognitive function, few scholars have analyzed it. Studies have shown that in the abstract of English language academic papers, in the theme system, it is necessary to use a simple theme, rarely use multiple themes, often use conjunctions, anaphora, and repeated cohesive means, if a large number of conjunctions are applied, the anaphora of the concept and the pragmatic reasoning can be realized, then a unified cognitive world will be formed, and the degree of coherence of such texts is also very high.
\end{abstract}

\section{Introduction}

From the reader's point of view, although the "reader" belongs to the group concept, it is composed of individuals. The individual has group characteristics and also has its own individual characteristics and cognitive perspective. When the cognitive perspective and the discourse coherence are related, different readers will have their own interpretations in the same discourse, and then form a discourse coherence with differences. From the perspective of cognitive theory and the influence of coherent interpretation of discourse, the reason for this problem is due to the influence of cognitive framework conversion factors. Discourse coherence belongs to the meaning of the concepts of discourse, discourse construction and image schema, which are activated in the framework of language cognition. The discourse coherence has multiple levels, and the cognitive framework has many levels. Dimensions, although there are more or less differences in the cognitive framework model, are all tools for people to understand the world. Under normal circumstances, the source of the text is very much. It can cite other people's words. It can also be used to generate new texts. The quotations and retellings are all about transforming one's words into another. The language of behavior, and a different foothold. In addition, the facts expressed in the discourse are not completely objective, and they are often described from their own subjective perspectives. Each individual has a different perspective on the perspective of things, and then there will be different manifestations in the discourse.

With his own individual characteristics and cognitive perspective. When the cognitive perspective and the discourse coherence are related, different readers will have their own interpretations in the same discourse, and then form a discourse coherence with differences. From the perspective of cognitive theory and the influence of coherent interpretation of discourse, the reason for this problem is due to the influence of cognitive framework conversion factors. Discourse coherence belongs to the meaning of the concepts of discourse, discourse construction and image schema, which are activated in the framework of language cognition. The discourse coherence has multiple levels, and the cognitive framework has many levels. Dimensions, although there are more or less differences in the cognitive framework model, are all tools for people to understand the world. Under normal circumstances, the source of the text is very much. It can cite other people's words. It can also be used to generate new texts. The quotations and retellings are all about transforming one's words into another. The language of behavior, and a different foothold. In addition, the facts expressed in the discourse are not completely objective, and they are often described from their own subjective perspectives. Each individual has a different perspective on the perspective of things, and then there will be different manifestations in the discourse. The language 
construction is a combination of semantics, syntax, pragmatics and phonology. This is not only a requirement of language, but also a requirement of cognitive psychology for integrity. If the discourse construction is used as a discourse Structure, then you must define it accurately. In the process of discourse analysis, it is necessary to analyze the contextual factors and commonality of words and sentences, and take social and cultural factors related to them seriously. It can be said that discourse is a syntactic combination. There are also several exceptions: the first is the combination of sentences and complex sentences; the second is from the language category and text type as the starting point, analyzing the relationship between discourse knowledge and text structure. If the discourse construction is compared with the sentence hierarchy, then the meaning, function and form of the text and the discourse belong to the category of the structure. Discourse construction belongs to abstract entities and belongs to the framework of cognitive discourse structure. Among them, text type and language category are the main concepts. In recent years, with the deepening of people's research on discourse knowledge, people began to "text." Types and genre are divided into two different modules. Of course, both are still part of the macro-cognitive framework.

The representation of discourse construction includes three categories. The first is to place the discourse construction features in the macroscopic cognitive framework of the sentence. The second is to place the discourse construction in a cognitive framework that can enclose the sentence hierarchy. The third category is to treat the discourse construct as an independent content. For structural formulas, both their functions and meanings contain multiple dimensions, each of which has unique internal adjustments. Therefore, discourse construction is an extremely important textual coherent recognition structure.

\section{English linguistics academic paper abstract cognitive function in textual coherence}

Under the framework of cognitive theory, the focus of people's research is on the dynamic operation of people's psychology. The discourses produced in this context have coherent characteristics. The theory of cognition coherence can not only coherence of dialogue. To answer, we can also support our research below. With regard to the abstracts of academic papers in English linguistics, it is a concept of compression. Each discourse has important meanings. In the abstract of academic papers, it can be divided into two parts: information abstract and statement abstract. The abstract emphasizes the results and conclusions. Reading abstracts, people generally understand the main meaning of an article. A completed abstract usually includes four parts: purpose, method, result, and conclusion. Although these elements do not necessarily all appear in the abstract, they need to be strictly expressed. The main meaning of the article, and in order to construct the required text relationship, you can use the clause complex according to the type of the article. According to the economic principle of the abstract, when writing, it is necessary to avoid repetitive words as much as possible, and use alternative or ellipsis to express them.

[Abstract] Multiple dependencies and the role of the grammar in real-time comprehension (1)Wh-dependencies are known to be formed rapidly in real-time comprehension. (2)The parser posits the location of gap sites in advance of the bottom-up evidence for missing constituents, and must therefore have a means of deciding when and where to project dependencies. (3) Previous studies have observed that the parser avoids building ungrammatical wh-dependencies, for example, by restricting the search for gap sites from island domains. (4) This paper tests the stronger claim that constraints are not merely respected, but that grammatical knowledge actively prompts the construction of some representations in advance of the input. (5) Three self-paced reading experiments examined patterns of wh -dependency formation in multiple-dependency constructions: obligatory across-the-board (ATB) extraction from coordinated verb phrase . S, and from optional parasitic gaps in post-verbal adjunct clauses (6) The key finding is that comprehenders immediately enforce the requirement for extraction from coordinates, and hence actively search for multiple gap

sites within a coordinate VP; but they do not search for post-verbal parasitic gaps. (7) This difference can not be attributed to relative differences in acceptability, as comprehenders rated both of these multiple-gap constructions equally highly, nor can it be explained by general parsing 
incentives to develop maximal incremental interpretations of partial (8) More plausibly, the difference reflects the deployment of detailed grammatical knowledge in a parser that is motivated to satisfy structural licensing requirements in real time.

Obviously, lexical cohesion is the main device of discourse organization in the abstract. The understanding of a discourse is determined by something else. If the determinant is verbally explicit, the discourse is cohesive. In this abstract, repetition appears to be the most important resource It has a reinforcing effect on the reader. What follows is a display of the results of repetition analysis. The order of the clauses is described in Arabic numerals:

\section{Cognitive Analysis of Discourse Coherence}

In cognitive linguistics, the lexical items, word categories and grammatical constructions are treated as conceptual categories and reflections of general cognitive principles instead of pure linguistic principles. The cognitive theories like dealized cognitive world, prototype theory and schema theory are decisive for the interpretation of mental coherence and the degree of discourse coherence.

In communication, people get access to the relations among statements and understand the whole discourse with the help of the cognitive world knowledge. A cognitive world is made by the combination of the ICM and the background information. Since Lakoff proposed in 1987 that the ICM should be used to explain the semantic categories and the conceptual structures, the ICM has become a very important part of cognitive linguistics. The ICM refers to the abstract, consistent and idealized understanding about the experience and knowledge in a particular field; it includes the widely accepted and conventional cognition models and language rules. To analyze discourse coherence in the sense of the cognitive world, we mainly rely on the information provided by the statements and people's own cognitive knowledge about the world. The ICM describes the coherence according to the following two principles: firstly, the lexical items form a unified cognitive world in reader's mind. Then the cognitive world serves as a cognitive reference point for the connection of the whole discourse.

When a certain micro-ICM is activated by a word, the information makes the whole discourse meaningful and supplements the default information. Take Abstract 1 for example:

[Abstract] A holistic approach to task-based interaction This paper proposes that interaction generated by tasks has previously been very difficult to analyze because of its highly indexical nature. Task-related actions and non-verbal communication could not be related easily to talk. A technological solution to this problem is presented, using a combination of task-tracking hardware and software, video recording and transcription. This enables a holistic approach, i.e. one in which all elements of behavior can be integrated in analysis. Micro-analyses of multimodel data are undertaken, which provide revealing insights into the processes of task-based learning. A framework for describing and analyzing task-based interaction from a holistic perspective is outlined.

"The problem" in the abstract activates an ICM about the problem: when referring to a problem, it means that there is something difficult to solve; according to our common sense, if there is a problem, we should resolve it; and to resolve a problem, we must know what the problem is. In this context, we need to pay special attention to the contents stated before: because the reciprocal action caused by the tasks is indexical, and it is difficult to analyze. However, we can not establish connection between the actions of the task and the communication easily except the verbal method. This forms a problem and it needs further discussion and resolution in the following part. Therefore, we should take into consideration the micro cognitive world in order to establish coherence.

[Abstract] The genre(s) of student writing: developing writing models This paper addresses the need to re-evaluate the aims and objectives underlying the teaching of English for Academic Purposes (EAP) in a European context. I argue here that for students to develop fully their abilities as writers, the objectives set in individual classes must reflect students' communicative purposes, rather than those of expert writers, and for that reason specific student writing models are needed. This paper proposes a methodology for the development of such writing models, and presents 
results of the application of this methodology to the analysis of a corpus of 55 student paper conclusions. The notion of student genre(s) adopted here challenges the tacit assumption that EAP is a homogeneous whole, and that expert models can realistically be used as models in teaching EAP student writing.

In the above text, the pronoun "those" is a reference in grammar and it represents some information previously mentioned. The phrase "for that reason" is a substitution and it omits the detailed description. It seems to be lack of cohesive relations in the linguistic form. However, we can supplement the information by inference with the help of the cognitive world knowledge. It is found that the pronoun "those" refers to "the objectives" and the pronoun "for that reason" refers to the attributive clause "for students to develop fully their abilities as writers, the objectives set in individual classes must reflect students' communicative purposes, rather than those of expert writers". The activation and supplementation of default information in the ICM is an important and practical method for the discourse coherence analysis.

\section{Conclusion}

Cognitive mode is a complementary discourse coherence function model. We believe that the ideal cognitive mode of English linguistics academic abstracts is to refer to the corresponding cohesive means and reference schema. Due to the limitation of space, we can not discourse. The coherent mechanism has been explored in depth, and some issues have yet to be further explored.

\section{References}

[1] Johnson, M. and G. Lakoff. Why Linguistics Requires Embodied Realism [J]. Cognitive Linguistics. 2002.

[2] Xiao Zhonghua, Cao Yan. A comparative study of multi-dimensional language steps in English abstracts of Chinese and foreign authors' scientific papers [J]. Foreign Language Teaching and Research, 2014(2).

[3] Yan Yumei. Genre analysis and abstract discourse of English and Chinese academic papers [J]. Foreign Language Teaching, 2004 (2).

[4] Zofia Golebiowski. Prominent messages in Education and Applied Linguistic abstracts: How do authors appeal to their prospective readers?[J] . Journal of Pragmatics, 2008 (4).

[5] Zhang Delu. Form and meaning in textual cohesion [J]. Foreign Languages (Journal of Shanghai International Studies University), 2005 (5) 